\title{
Assessment of Spatial Distribution and Estimation of Biomass of Prosopis juliflora (Sw.) DC. in Puttlam to Mannar Region of Sri Lanka using Remote Sensing and GIS
}

\author{
A.R. Gunawardena, T.T. Fernando, S.P. Nissanka ${ }^{1 *}$ and N.D.K. Dayawansa ${ }^{2}$ \\ Postgraduate Institute of Agriculture \\ University of Peradeniya \\ Sri Lanka
}

\begin{abstract}
Prosopis juliflora (Sw.) DC. has been introduced to Hambantota District in the southern province of Sri Lanka during early 1950's to rehabilitate salt affected soils in the coastal area and for firewood purposes. Within a very short period of time, this species got naturalized and started to show aggressive growing pattern. Currently, it covers almost all semi-arid areas including north-western coast of Sri Lanka. This study was conducted to identify the spatial distribution, to estimate the biomass and to map the vulnerable areas of spreading of $\underline{P}$. juliflora in Puttalam to Mannar region of Sri Lanka using remote sensing and geographic information system (GIS). Landsat ETM+ (2005), ALOS AVNIR (2010) and GeoEye (2012) satellite data were used for the study. The existing and past situation of the distribution of this invasive species was determined using supervised and unsupervised image classification, developing NDVI and by visual interpretation. Above ground live biomass was observed through NDVI with allometric equations. A GIS-based model was developed to identify the vulnerable areas for spreading of the species. This study identified that visual interpretation of high spatial resolution satellite data is the most ideal in identification of the areas infested with $\underline{P}$. juliflora. Further it was identified that a combination of supervised and unsupervised classification of medium spatial resolution satellite data and NDVI provides reasonable results in identifying $\underline{P}$. juliflora infested areas with an accuracy of $78 \%$. The results revealed that the biomass content associated with $\underline{P}$. juliflora-associated vegetation was higher in 2010 compared to 2005 indicating spreading of the species. Vulnerable area mapping is helpful in controlling further spreading of the species. This study proves the importance and effectiveness of remote sensing and GIS in identification and assessment of areas infested with $\underline{P}$.juliflora and their spatial spreading patterns.
\end{abstract}

Keywords: biomass, Invasive species, remote sensing, satellite images, spatial distribution

\section{INTRODUCTION}

Invasive plants are plants that have been introduced into an environment in which they did not evolve and thus usually have no natural enemies to limit their production and spreading patterns (McQueen \& Noemdoe, 2000).

1 Department of Crop Science, Faculty of Agriculture, University of Peradeniya, Sri Lanka

2 Department of Agricultural Engineering, Faculty of Agriculture, University of Peradeniya, Sri Lanka

Corresponding author: spn@pdn.ac.lk 
At the global scale, invasive species have been identified as one of the main reasons of biodiversity loss and substantial economic damage. A large number of studies have therefore been conducted investigating species invasions with the aim to identify suitable habitats and to formulate coordinated management plans. The findings of these studies contribute to early detection and elimination of invasive species to reduce their negative impacts.

Fast growth, drought resistance and with remarkable coppicing power, Prosopis is a natural fuel wood candidate. This species provides $>90 \%$ of the fuel wood in some Indian villages (Sharma, 1981). Although no direct data on N-fixation of Prosopis are available, Felker \& Bandurski (1979) suggested that tree legumes (exclusive of Caesalpiniaceae) fix between 155 and $580 \mathrm{~kg} / \mathrm{ha} / \mathrm{yr}$. The nature of adaptation to harsh environment conditions helps the species to spread over the areas depressing the native species. Mean annual temperature between $5-34^{\circ} \mathrm{C}$, rainfall between 50- $1200 \mathrm{~mm}$, elevation below $1500 \mathrm{~m}$ are the biophysical limits of the species. It can grow on a variety of soils including rocky hills, saline flats, on shifting sand dunes and coastal sand, although it attains its best size in localities protected from wind and having the water table not far below the surface. It can grow in waterlogged conditions and is tolerant to high salinity.

P. juliflora has been cultivated in arid areas of tropical and subtropical regions in Africa and Asia under the aforestation programs and it has been introduced to Hambantota District in the Southern province of Sri Lanka during the early 1950's to rehabilitate salt affected soils and for firewood. Within a very short period of time, this species got naturalized and started showing aggressive growing pattern and currently it covers almost all areas of the Bundala National Park depressing the regeneration of native plant species. As an island, Sri Lanka has a large number of lagoons and coastal areas which are ideal ecosystems to spread P. juliflora. Among them, Puttalam to Mannar coastal area of Sri Lanka is highly vulnerable to spreading of this species.

Mapping of invasive species provides baseline information which is essential to assess the spreading pattern while offering a great platform to educate the public, inform decision makers, solicit support, and to document the progress of management measures implemented. Remote sensing and GIS can be effectively utilized for this purpose. The objective of this study was to investigate the spatial distribution, abundance, production status, habitat preferences, and rate of invasiveness of $P$. juliflora and vulnerable areas of spreading the species in Puttlam to Mannar region of Sri Lanka using remote sensing and GIS techniques.

\section{METHODOLOGY}

\section{Study Area}

This study was carried out in 2011 in selected 07 divisional secretariat divisions in Puttalam to Mannar coastal region. Most of the selected areas were covered with marshy land, scrubland and paddy which are highly vulnerable to spreading of P. juliflora. The location map is shown in Fig. 1. 


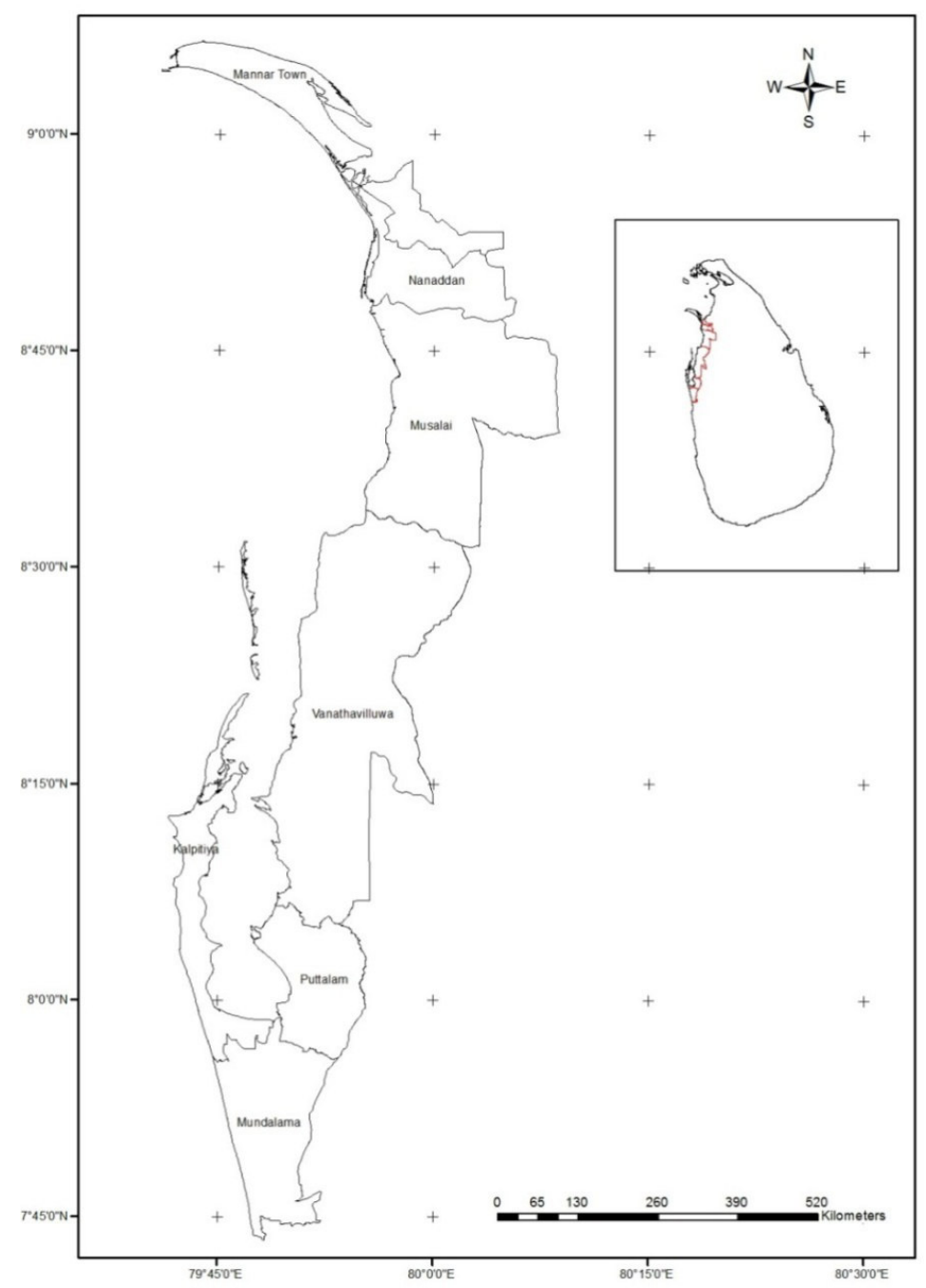

Fig. 1. Location map of the study area

\section{Data, Materials and other Information}

Landsat ETM+ (2005), ALOS AVNIR (2010), GeoEye (2013) satellite data were used for the study to prepare land use/ land cover maps and to identify the invasive species.

Vector data of 1: 50,000 and 10,000 scale topographic maps from the Survey Department were used for satellite data interpretation and land use/ cover identification.

Intensive field work was carried out during January to December 2012 in the study area. Accordingly, 164 sampling plots $(30 \times 30 \mathrm{~m})$ were used to collect detailed information on vegetation (canopy cover, density, species type, DBH and heights), topography and soil properties (moisture, slope, exposure). GPS locations of each sampling plots were obtained. Digital laser clinometer and DBH tape were used to collect data on trees. 


\section{Visual interpretation of high resolution satellite data}

Visual and digital image interpretation techniques were used to identify the land use and land cover information in the study area in order to identify the locations where the invasive species are distributed. Visual interpretation was done with the high resolution (GeoEye2012) satellite image and manual digitizing was applied to identify land use/ cover. Land use/ cover types identified by this methods was verified with the ground truth data collected using GPS. Though this method is high in accuracy, due to time consuming nature, it was applied only to a few selected sampling locations.

\section{Digital image interpretation}

Landsat 7 ETM+ (2005) image digital numbers were converted to reflectance using the method outlined in the Landsat 7 Science Data Users Handbook (2004). Since the gain on each of the Landsat 7 ETM+ channels can vary from high to low between successive overpasses, it is essential to convert apparent digital numbers to reflectance for consistency between different bands. Acquired images were originally geo-referenced to UTM (Universal Transverse Mercator) projection. Subsequently, all the medium resolution satellite images (Landsat ETM+ and AVNIR) were subjected to supervised classification with the aid of collected ground truth data.

\section{Development of Normalized Difference Vegetation Index (NDVI) images}

Vegetation indices are commonly used in remote sensing for assessment of vegetation characteristics. Among the indices, Normalized Difference Vegetation Index (NDVI) is the most widely used index for vegetation monitoring with respect to biomass calculation, measure leaf area index, yield estimation and to monitor vegetation vigor (Panda, 2005). NDVI was developed for the satellite images used in this study using the formula given in Equation 1.

$$
\mathrm{NDVI}=(\mathrm{NIR}-\mathrm{Red}) /(\mathrm{NIR}+\mathrm{Red}) \quad \text { Equation } 1 .
$$

Landsat ETM+ and ALOS AVNIR band combination for NDVI generation is given in Equation 2.

$$
\mathrm{NDVI}=(\text { Band } 4-\text { Band } 3) /(\text { Band } 4+\text { Band3 }) \quad \text { Equation } 2 .
$$

Finally, the temporal distribution of the invasive plant species was identified and the area extents were calculated.

\section{Measurement of live biomass using remote sensing}

Changes of above ground live biomass is a worthy indicator to estimate spreading rate of $P$. juliflora and therefore biomass was directly estimated through the previously derived relationships between remote sensing based vegetation indices (Brown et al., 2004). This method was utilized to assess the changes of biomass of $P$. juliflora and associated species and to identify the spreading areas during 2005 to 2010 using Landsat ETM+ and AVNIR satellite images. The recommended equations to estimate biomass for shrubs and small tree species (Equation 3 ) were used to estimate the biomass of $P$. juliflora in the study area. 
A criteria was developed to identify the possibility of spreading of $P$. Juliflora using following parameters.

- Invasion threat is high for the areas within $1 \mathrm{~km}$ distance from the $P$. Juliflora rich areas.

- Invasion threat is high in areas which are closer to the water ways which run through $P$. Juliflora rich areas. This threat can go upto $5 \mathrm{~km}$ distance from the streams/ rivers.

- Invasion threat is high in grasslands used for cattle grazing which are within $5 \mathrm{~km}$ distance from the P. Juliflora rich areas.

- Invasion threat is high in possible sites of soil excavation, transportation routes, land development sites.

- Invasion threat is high in paddy lands irrigated from water coming through the invasive plant distributed areas.

\section{RESULTS AND DISCUSSION}

\section{Identification of $P$. juliflora using visual interpretation of satellite data}

The visual interpretation of high spatial resolution GeoEye (2010) satellite images provided highly precise results in identification of $P$. juliflora. The size, color, shape, surface roughness, pattern of canopies and surrounding features were considered in identifying $P$. juliflora associated vegetation. It was found that there are some difficulties in differentiating mangroves and $P$. juliflora due to similar spectral characteristics. Ground truth information was very helpful in determining the accuracy of the output. Since this method is very time consuming, it is not possible to apply to a large spatial extent. Fig. 2 presents the results of the visual image interpretation. 


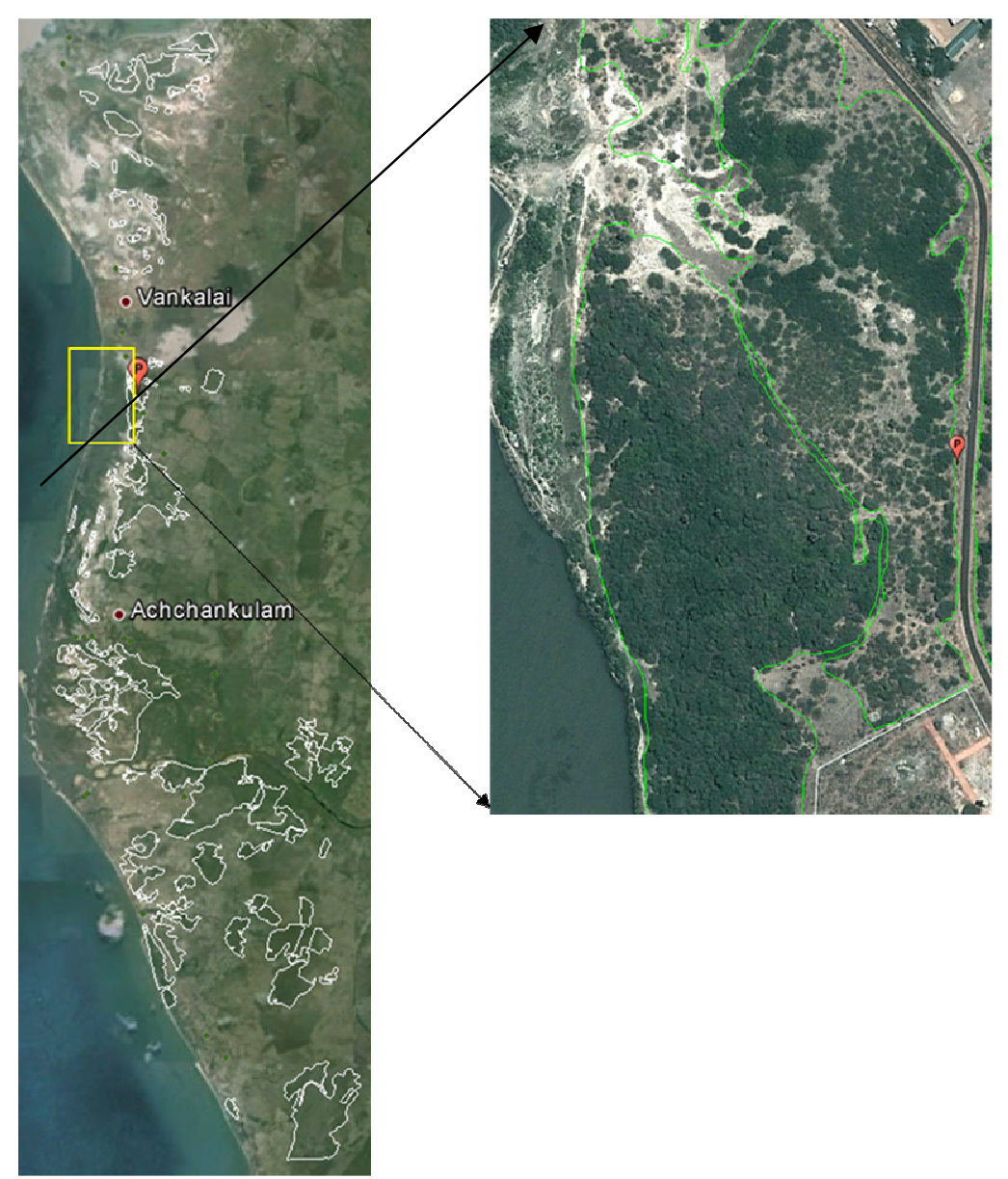

\section{Fig. 2. P. juliflora associated vegetation identified using visual interpretation of GeoEye (2012) high resolution satellite image.}

\section{Identification of $\boldsymbol{P}$. juliflora using digital image interpretation}

Twenty pixel clusters were identified using a combination of supervised and unsupervised classification methods of Landsat ETM+ (2005) satellite data. Spectral reflectance graphs were developed with the classified images. With the aid of the ground truth data, it was found that spectral classes 12 to 16 represent $P$. juliflora dominated vegetation in the unsupervised classification output (Fig. 3). 


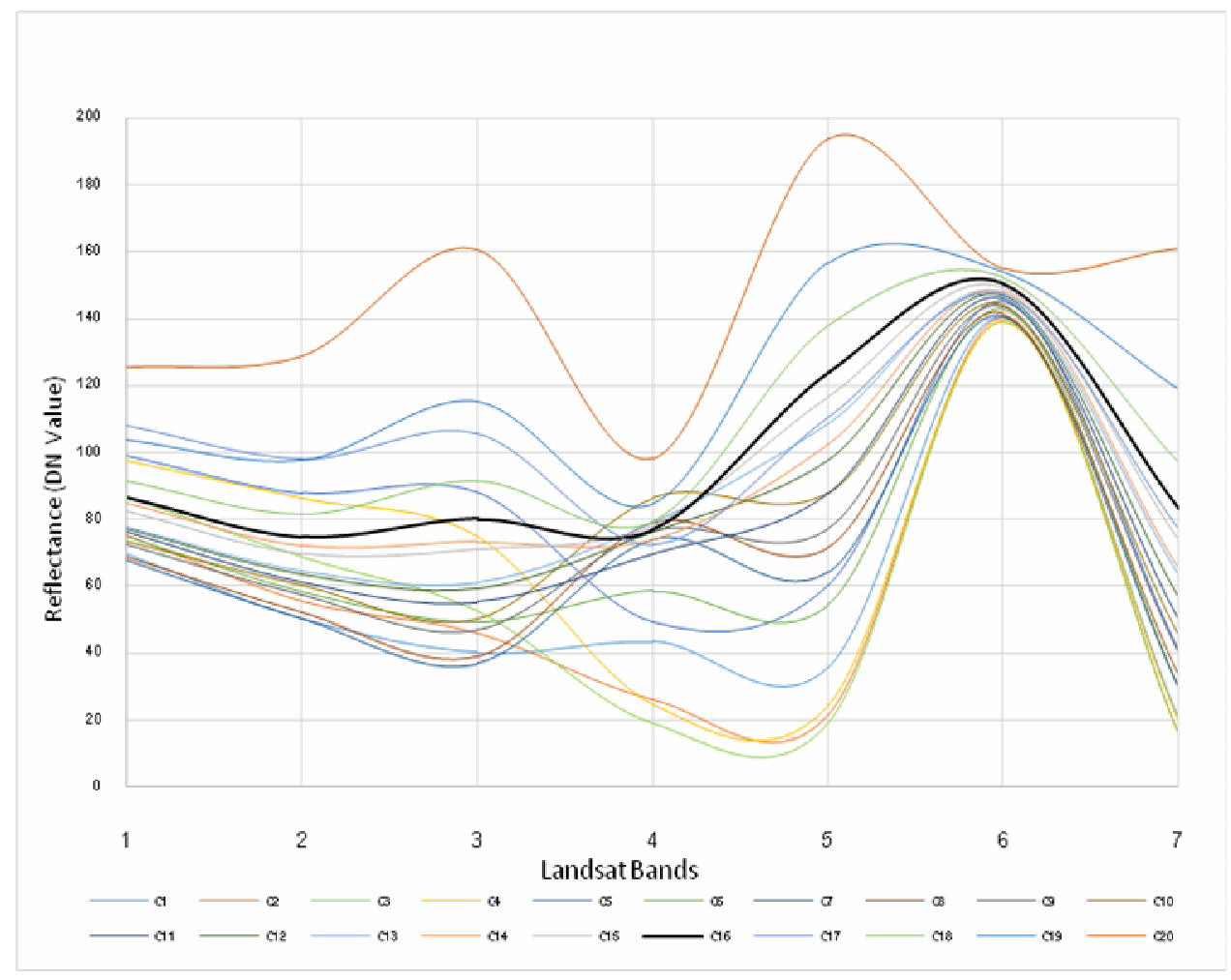

Fig. 3. Spectral reflectance curves of unsupervised 20-class image from Landsat ETM+ (2005) and classes 12-16 represent $P$. juliflora dominated vegetation.

Vegetation has a unique spectral signature which enables it to be distinguished readily from other type of land cover in an optical/near-infrared image. The reflectance is low in both blue and red regions of the spectrum, due to absorption by chlorophyll for photosynthesis. It has a peak at the green region. In the near infrared (NIR) region, the reflectance is much higher than that in the visible band due to the cellular structure in the leaves. Hence, vegetation can be identified by the high NIR but generally low visible reflectance. Reflectance increases with increasing wavelength (http://www.cps-amu.org/sf/notes/m1r-1-8.htm).

\section{The bi-spectral plots}

The bi-spectral plots derived from the red and near infrared bands of relevant satellite sensors also used to further verification of land cover types. Mean digital value of each class was plotted against the Red and Near Infrared (NIR) bands of two images separately to locate the greenness, wetness and brightness plots. The bi-spectral scatter plot is a very useful tool to observe spatial distribution of each land cover class and also, the plot can be used to group similar classes. Classes that occupy green area have high NIR reflectivity and low red reflectance. Typically, these areas are forests, agricultural lands, and natural vegetation. Classes that occupy bright areas have high NIR and high red reflectance (Densheng et al., 2004). The spatial location of invasive species associated vegetation is shown in graph just above the soil line mixed with water and moderately dense vegetation (Fig. 4). 


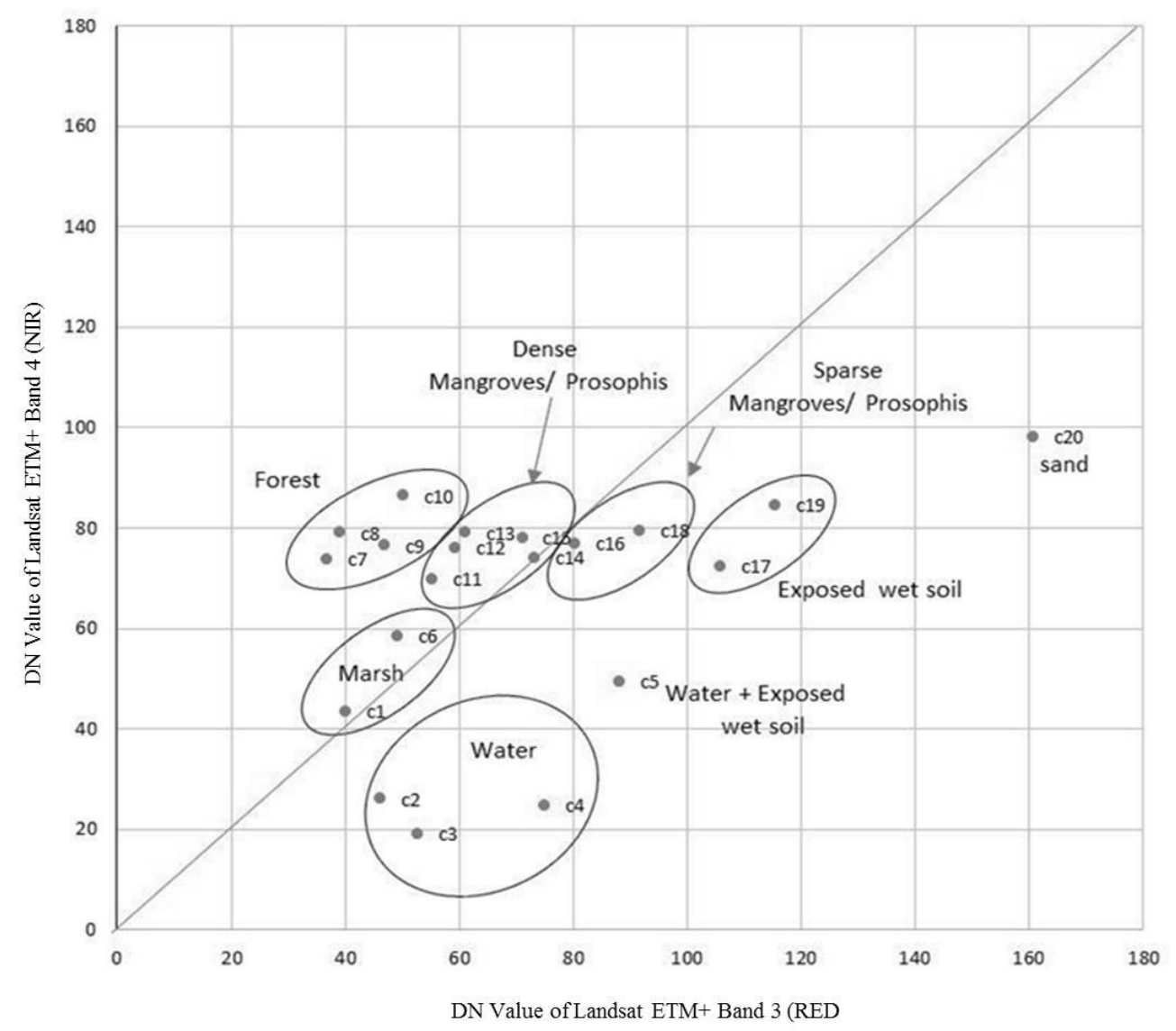

Fig. 4. Bi-spectral plots of supervised 20 class image from Landsat ETM+ (2005) showing distribution of scattered land cover classes.

\section{Feature space diagrams}

Feature spaces is one of the methods in remote sensing to observe class overlapping and this method was used for the verification of $P$. juliflora by red and NIR spectral reflectance in each satellite images (Fig. 5). According to the observation through feature spaces developed for both Landsat ETM + and AVNIR images, there are more overlapping of $P$. juliflora with scrub, other plantation and mangroves in ALOS-AVNIR image than that of Landsat ETM+ image. However, using feature spaces it was found that water, bare soil, thick vegetation, and sand dominated land cover types can be easily differentiate from invasive plants associated vegetation. 


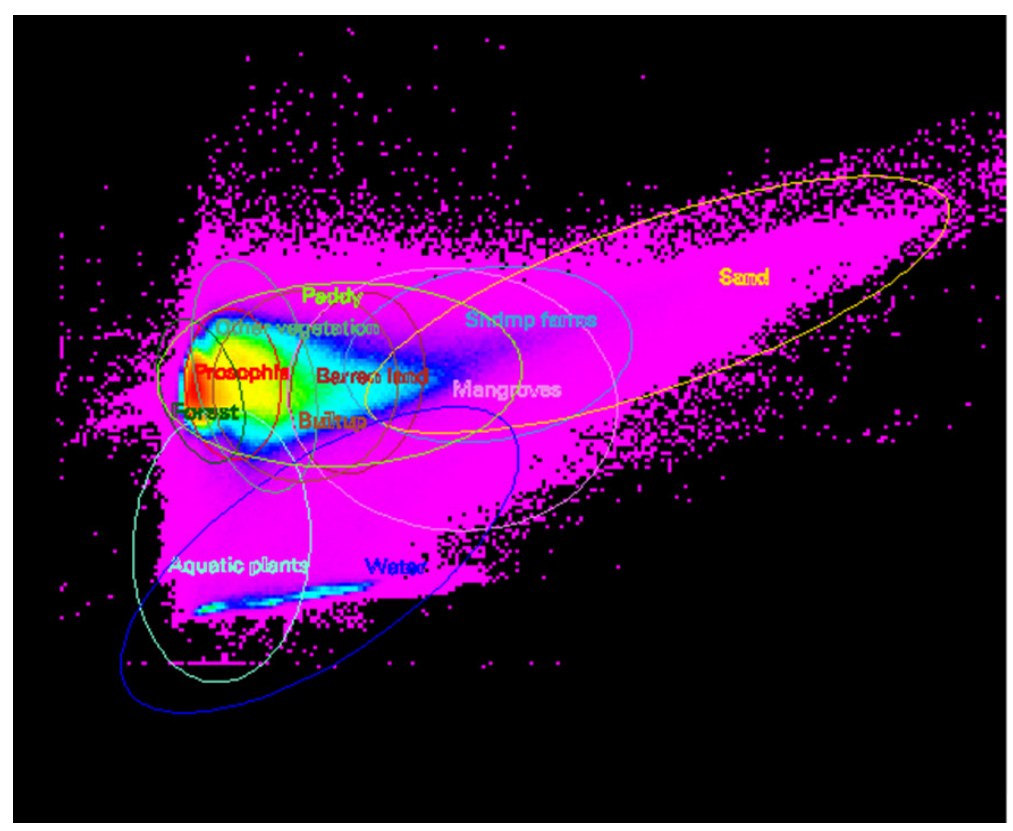

Fig. 5. Feature spaces developed for ALOS-AVNIR (2010) images to differentiate $P$. juliflora from other classes

There are some limitations and drawbacks observed in this method as selected two images have different sensor properties such as pixel resolution, spectral bands and radiometric incompatibilities. There were many limitations came across in identification of $P$. juliflora using image classification techniques. The main limitation identified was the mixed pixels. Hence, it was further attempted to use Normalized Difference Vegetation Index (NDVI) to identify $P$. juliflora.

\section{Normalized Difference Vegetation Index (NDVI) to identify $P$. juliflora}

NDVI is used to estimate the portion of photosynthetically active radiation absorbed by the crop canopy (Asrar et al., 1984). The prepared NDVI images were classified into 20 classes by re-coding of pixels. Fig. 6. presents the four land cover clusters identified by plotting the NDVI value in each class of ALOS-AVNIR image. These four classes are water, thick vegetation, moderately-dense vegetation and bare/ exposed soil. It was found that $P$. juliflora belongs to the category of moderately dense vegetation with high soil moisture and mixed with scrubs and mangroves. 


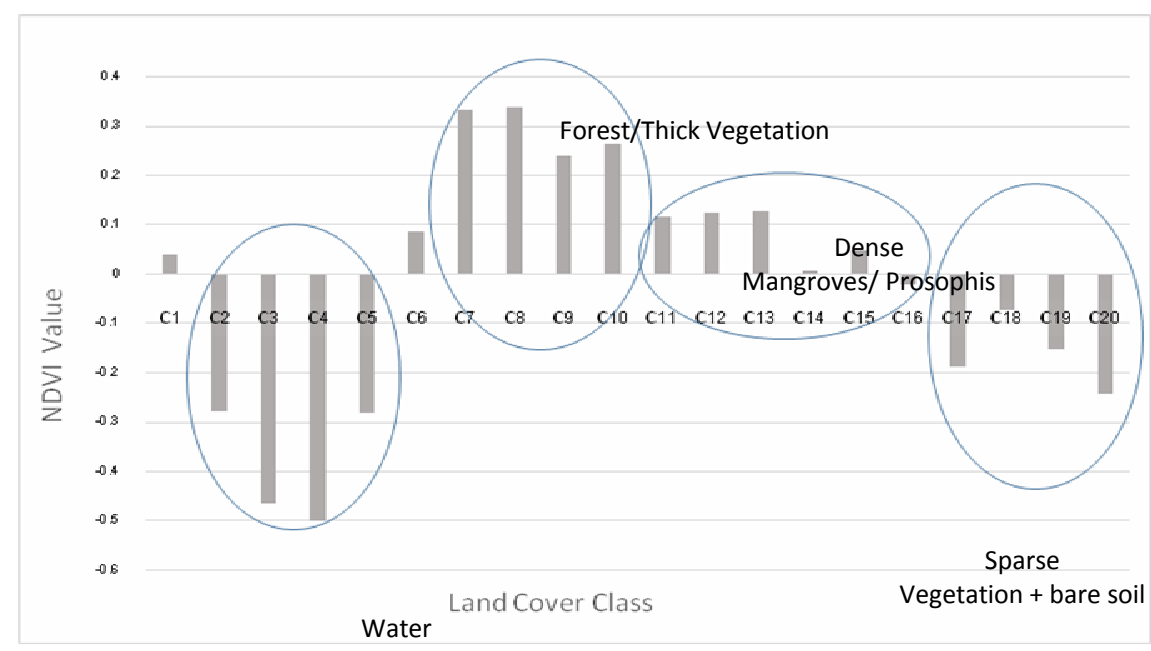

Fig. 6. The NDVI graph developed for ALOS-AVNIR (2010) image to identify P. juliflora associated vegetation

These NDVI graphs also verified the findings in supervised/ unsupervised classification that classes 12 to 16 as $P$. juliflora associated vegetation. This was further confirmed by the ground truth and visual interpretation results. The accuracy level of identifying $P$. juliflora associated vegetation is $78 \%$ according to Kappa statistics.

\section{Detection of distribution of $P$. juliflora with time}

Spatial distribution pattern of $P$. juliflora with time was assessed using Landsat ETM+ (2005) and ALOS-AVNIR (2010) satellite images.

Finally, it was found that there are five invasive species dominated land cover type as chena, scrubland, marshland, sand or bare land and grasses. Analysis found that there is a high level of $P$. juliflora dominated vegetation in 2010 compared to 2005 (Table 1).

Table. 1. $P$. juliflora association vegetation in 2005 and 2010

\begin{tabular}{lcr}
\hline \multicolumn{1}{c}{ Selected Land Cover Type } & Estimate area (ha) & \\
& 2005 & 2010 \\
\hline Prosophis mixed with Chena & 31.37 & 31.19 \\
Prosophis mixed with Grassland & 11.20 & 22.38 \\
Prosophis mixed with Marshland & 188.1 & 268.4 \\
Prosophis mixed with Sand/ bare land & 124.02 & 194.7 \\
Prosophis mixed with Scrubland & 1029.5 & 1966.82 \\
\hline
\end{tabular}


The results found the scrubland are the most threatened land cover types for invasion by $P$. juliflora. Marsh lands are also vulnerable to spreading of $P$. juliflora.

\section{Biomass estimation}

The results show an increment of biomass during the period of 2005 to 2010 as a result of rapid spreading of invasive species. The results of the calculated total biomass content needs further classification according to the land cover types. Estimation of biomass of $P$. juliflora is a challenging task due to its mixing with other land cover types.

\section{Vulnerable area mapping of $\boldsymbol{P}$. juliflora}

Fig. 7 presents the vulnerable areas identified using the criteria defined in the methodology. This map can be used as a tool in management of further spreading of $P$. juliflora.

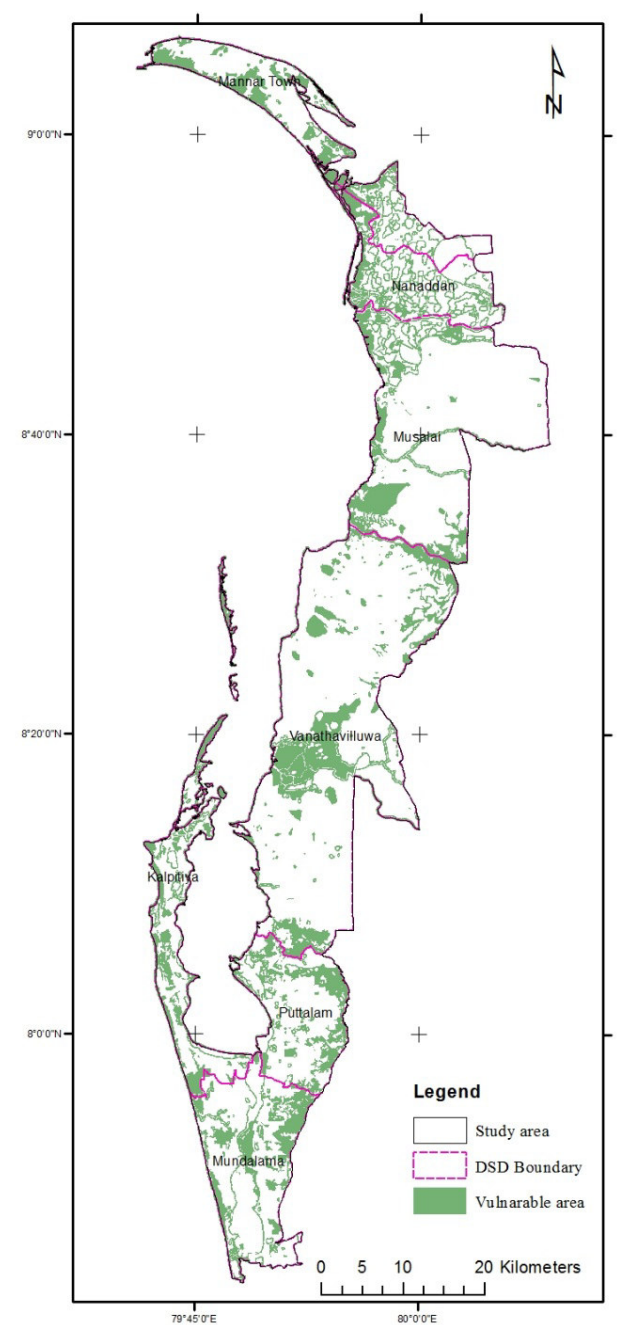

Fig. 7. Vulnerable areas for spreading of $P$. juliflora 


\section{CONCLUSIONS}

P. juliflora is one of the leading invasive species dominant in semi-arid regions of Sri Lanka. This study identified that visual interpretation of high spatial resolution satellite data is the most ideal in identification of the areas infested with $P$. juliflora. However, it has limitations due to high cost of images and time consumption for analysis. Study identified that a combination of pixel based supervised/ unsupervised classification of medium spatial resolution satellite data and NDVI provides a reasonable results in identifying $P$. juliflora infested areas. This provided an accuracy of $78 \%$ in identification of $P$. juliflora using ALOS-AVNIR data. Study revealed that the biomass content associated with $P$. juliflora associated vegetation is high in 2010 compared to 2005. This is an indication of spreading of this species.

The maps produced to identify possible vulnerable areas for spreading is an ideal tool in controlling further spreading of the species. However, proper identification of plant, climatic and anthropogenic parameters is necessary to develop a very accurate model to apply management interventions in more efficient manner.

This study proves the importance and effectiveness of remote Sensing and GIS in identification and assessment of areas infested with invasive plant species and their spatial spreading patterns.

\section{REFERENCES}

Aranha, J.T., Viana, H.F. and Rodrigues, R., (2008). Vegetation classification by satellite image processing, A case study in North Portugal. International Conference and Exhibition on Bioenergy, International Conference and Exhibition on Bioenergy, April 6th - 9th 2008, Universidade do Minho, Guimarães, Portugal.

Asrar, G., Fuchs M., Kanemasu, E.T. and Hatfield, J.L. (1984). Estimating absorbed photosynthetic radiation and leaf area index from spectral reflectance in wheat, Agron. J. 76, 300-306.

Brown, S.D., Shoch, T., Pearson and Delaney.M., (2004).Methods for Measuring and Monitoring Forestry Carbon Projects in California. Winrock International, for the California Energy Commission, PIER Energy related Environmental Research. 500-04-072F: 15 - 17.

Densheng, Lu., Mausel, P., Brondizio, E. and Moran, E., (2004). Relationship between forest stand parameters and Landsat TM spectral response in the Brazilian Amazon Basin, Forest Ecology and Management: 198, 149-167.

Felker, P. and Bandurski, R.S. (1979). Uses and potential uses of leguminous trees for minimal energy input agriculture. Economic Bot. 33, 172-184.

McQueen, C., \& Noemdoe, S. (2000). The Working for Water programme. Paper presented at the Best Management Practices for prevention and controlling Invasive Alien Species, Cape Town, South Africa.

Panda, B.C. (2005). Remote sensing principles and applications. Viva books Privet Limited, New Delhi. 100 - 148.

Sharma IK (1981). Ecological and Economic importance of Prosopis juliflora in the Indian Thar Desert. Journal of Taxonomy and Botany 2, 245-248. 